\title{
Calculation of lunar orbit anomaly
}

\author{
Louise Riofrio
}

\begin{abstract}
Background: Studies of the Moon, with thanks to NASA and Johnson Space Center, have quantified an anomaly in measurements of lunar orbital evolution. This finding may have significance for cosmology and the speed of light. The Lunar Laser Ranging Experiment from Apollo reports the Moon's semimajor axis increasing at a rate of $3.82 \pm .07 \mathrm{~cm} / \mathrm{yr}$, anomalously high.

Findings: Sedimentary data indicates a rate of only $2.9 \pm 0.6 \mathrm{~cm} / \mathrm{yr}$. From historical eclipse records we can accurately calculate a rate of $2.82 \pm .08 \mathrm{~cm} / \mathrm{yr}$. A detailed numerical simulation of lunar orbital evolution predicts $2.91 \mathrm{~cm} / \mathrm{yr}$. LLRE's laser light differs from independent experiments by up to 120 .

Conclusions: Several possible explanations are considered. The author's hypothesis proposes that the speed of light decreases at rate $\dot{c} / c=-0.24 \times 10^{-10} \mathrm{yr}^{-1}$. This predicts that LLRE will differ by $0.935 \mathrm{~cm} / \mathrm{yr}$, precisely accounting for the lunar anomaly.
\end{abstract}

Keywords: Cosmology, Laser ranging, Lunar orbit, Relativity, Speed of light

\section{Lunar laser ranging}

Quantities that appear constant may change over time. The Moon's orbital distance has long been known to be slowly increasing. Simultaneously Earth's rotation rate has been slowing, causing increase in length of day. This is interpreted as tidal forces transferring angular momentum from Earth to Moon, causing the orbital semimajor axis to increase. Study of lunar orbital evolution draws data from multiple sources including sediments, eclipse observations and numerical simulation. Results from the Moon have implications for cosmology and light.

The Lunar Laser Ranging Experiment bounces laser light off corner reflectors placed on the Moon's surface. Reflectors were left behind by the Apollo 11, 14, 15 and Lunokhod missions. LLRE has been used to investigate geophysics of the Earth-Moon system and test Relativity's equivalence principle. Accuracy has been considered fine enough to rule out significant changes in the gravitational constant $G$ [1-5].

LLRE has measured the Moon's orbital semimajor axis $a=384,402 \mathrm{~km}$. Repeated measurements by LLRE over

Correspondence: Sailorstarfightr@yahoo.com

2004 San Sebastian Court, Houston, USA decades, as compiled by Dickey and colleagues, [6] appear to indicate that distance increasing at rate:

$$
\dot{a}=3.82 \pm .07 \mathrm{~cm} / \mathrm{yr}
$$

Here $\dot{a}$ is derivative of lunar orbital semimajor axis.

This value of $\dot{a}$ has been described as anomalously high [7]. As calculated by Bills and Ray, if the Moon were today gaining angular momentum at this rate it would have coincided with Earth about 1.5 Gyr ago. Studies of lunar samples show that the Moon has existed separately from Earth for over 4.5 Gyr. Today the LLRE measurement can be compared with independent experiments, with surprising results.

\section{Tidal rhythmites}

Geology and paleontology can also tell how the Moon's orbit has changed. Tidal rhythmites, in particular, carry a record of lunar-induced tides. Rhythmites leave layers on a daily and monthly frequency. Thicknesses of sedimentary layers vary with the height of local tides. As reviewed by Bills and Ray, fossilized rhythmites can determine lunar orbital distance millions of years in the past.

The Mansfield sediment of Indiana, the most recent and accurate to be studied, places the Moon 375,300 \pm 
$1,900 \mathrm{~km}$ away at a time $310 \mathrm{Myr}$ ago. For an average recession rate over this period, I subtract from today's distance and divide by time:

$$
\begin{aligned}
\dot{a} & =\frac{384,402 \mathrm{~km}-(375,300 \pm 1,900 \mathrm{~km})}{310 M y r} \\
& =2.9 \pm 0.6 \mathrm{~cm} / \mathrm{yr}
\end{aligned}
$$

Independent study by Williams [8] of the older Elatina and Reynella tidal rhythmites also indicates a lower recession rate than LLRE.

\section{Eclipse records}

Corroborating data may have come from historical astronomers. If the narrow track of total eclipse has been reported over an observatory, it provides an accurate measure of Earth's slowing rotation rate. When Earth and Moon are considered as a closed system, this tells us how much angular momentum has been transferred between them. For determining lunar recession rate, accuracy of this method can complement LLRE.

Lunar recession rate is thought to vary with change in Earth rotation period. A rate of $\dot{a}=3.82 \pm .07 \mathrm{~cm} / \mathrm{yr}$ corresponds to change in Earth's length of day of $2.30 \mathrm{msec} / \mathrm{cyr}$. Observations spanning 2700 yrs compiled by Stephenson and Morrison [9] show change in LOD of $1.70 \pm .05 \mathrm{msec} / \mathrm{cyr}$.

When change in LOD varies linearly this author calculates, incorporating the $3 \%$ standard deviation:

$$
\begin{aligned}
& \frac{\dot{a}}{1.70 \pm .05 m \mathrm{sec} / \mathrm{cyr}}=\frac{3.82 \pm .07 \mathrm{~cm} / y r}{2.30 m \mathrm{sec} / \mathrm{cyr}} \\
& \dot{a}=2.82 \pm .08 \mathrm{~cm} / y r
\end{aligned}
$$

Though eclipse records corroborate Mansfield tidal data, LLRE's laser light differs by over $12 \sigma$.

\section{Numerical simulation}

Transfer of angular momentum from Earth to Moon is subject to many factors. These include height of local tides, ocean depth, location of ocean basins and the slow movement of continental plates. Simple models often fail to account for all these influences. Poliakow [10] has produced a comprehensive numerical simulation of Earth-Moon tidal evolution. The model has been successfully used to solve the independent problem of predicting Earth tides, and may be considered highly robust. In the tidal model, some quantities usually taken as constant become variables.

The simulation predicts for the present:

$$
\dot{a}=2.91 \mathrm{~cm} / \mathrm{yr}
$$

A lower lunar recession rate $\dot{a}$, from three independent datasets, is extremely robust.

\section{Possible explanations}

Different sources have been proposed for lunar orbit anomalies. The lower recession rate found in sedimentary data has been attributed to increasing tides over millions of years. This inference has not been independently verified. For the Moon's recession to vary so greatly, tidal heights would have to increase enormously over time. Mansfield and other sediments do not show significantly different tidal heights than today.

The recession rate found from eclipse records has been suggested to result from glacial isostatic compensation. This is also an inference, for a very large change would again be required. Glacial reports are also available, and the extent of Earth glaciation over 2700 years has not changed in the amounts required. Sea-level studies suggest that tidal friction has not changed appreciably over this time [11].

These first two explanations may be mutually exclusive. Anomalous tidal changes over millions of years may not explain an anomaly in records just 2700 years old. Conversely, the inference of glacial change could not be maintained over the 310 Gyr lifespan of the youngest Mansfield sediments. The numerical simulation takes into account many factors influencing lunar recession rate, and agrees with experiment. As the lower recession rate is found in three completely independent datasets, alternate explanations must be considered.

Unknown accelerative components may also be considered to affect lunar orbit. Recession rate could then vary non-linearly with change in Earth LOD. An unknown amount of angular momentum may be transferred to the rotational component of the Moon's momentum. This would likely be a small effect, as the Moon's mass is large. Such an effect would also result in a lower recession rate, compounding the anomaly.

We may also consider unknown components in Earth's rotational momentum. If a portion of Earth's interior were rotating at a faster rate, angular momentum available for transfer to the Moon would be increased. Rapidly rotating regions within the core form a fascinating research subject. However, the inner Earth may not undergo the surface tides to transfer momentum. While a rapidly rotating inner element is an interesting research subject, it may not contribute to the orbital anomaly.

\section{Cosmological origin}

Anomalies in orbital measurements of Mercury and the moons of Jupiter are today known to result from Relativity and the speed of light $c$. A discrepancy of 43 " / cyr in Mercury's 5600"precession is a sign of General Relativity. An anomaly in observations of Jupiter's moons was predicted by Roemer from the finite speed of light. 
All possibilities being considered, the lunar anomaly may have a cosmological origin.

This author's hypothesis may be summarized simply:

$$
G M=t c^{3}
$$

where $t$ is age of the Universe, GM is the gravitational constant multiplied by a constant with dimensions of mass.

Speed of light $c$ would then be given by:

$$
\begin{aligned}
& c(t)=(G M)^{1 / 3} t^{-1 / 3} \\
& \dot{c}(t)=(-1 / 3)(G M)^{1 / 3} t^{-4 / 3} \\
& \frac{\dot{c}}{c}=-\frac{1}{3 t}=-0.24 \times 10^{-10} y r^{-1}
\end{aligned}
$$

where age of Universe $t$ is estimated at 13.7Gyr, the constants GM cancel.

By theory, when $t$ was small $c$ was enormous and the Universe would have expanded like a "Bang." As age $t$ increases, $c$ would slow due to gravitation and continue to slow at a tiny rate today. This model has been suggested to precisely fit the non-linear redshifts of distant Type Ia supernovae [12], the $4.507034 \%$ proportion of bayons and other puzzles. Cosmology makes a surprising but testable prediction: Time for laser light to return would increase each year, making the Moon appear to recede faster as measured by LLRE.

Apparent lunar distance would increase proportional to decrease in $c$ :

$$
\begin{aligned}
& \frac{\dot{a}}{a}=-\frac{\dot{c}}{c}=\frac{1}{3 t} \\
& \dot{a}=\frac{a}{3 t}=\frac{384,400 \mathrm{~km}}{3(13.7 G y r))} \\
& \dot{a}=0.935 \mathrm{~cm} / \mathrm{yr}
\end{aligned}
$$

where age of Universe $t$ is estimated at $13.7 \mathrm{Gyr}$, apparent distance is predicted to increase an additional $0.935 \mathrm{~cm} / y \mathrm{r}$, precisely accounting for the anomaly.

From LLRE and accounting for the speed of light, actual recession rate would be:

$$
\begin{aligned}
& \dot{a}=3.82 \pm .07 \mathrm{~cm} / y r-0.935 \mathrm{~cm} / y r \\
& \dot{a}=2.88 \pm .07 \mathrm{~cm} / y r
\end{aligned}
$$

This value is in $1 \sigma$ agreement with eclipse records, Mansfield sedimentary data and numerical simulation. If one of these three datasets were found to contain error, the other two would agree with prediction.

Variation of $\dot{c} / c=0.24 \times 10^{-10} \mathrm{yr}^{-1}$ equals $-0.72 \mathrm{~cm} / \mathrm{sec}$ $y r$, too small to have been detected by previous experiments. For example, a survey by Iorio [13] using data on planetary orbits limits cdot/c to $(0.5 \pm 2) \times 10^{-7} y r^{-1}$, more than 3 orders of magnitude greater. Iorio [14] has also written about a change in lunar orbital eccentricity. A change in semimajor axis could be interpreted as a change in eccentricity. However as lunar orbital angular momentum increases the semimajor and semiminor axes would both change proportionately. Iorio finds no known physical basis for an eccentricity change.

An apparent change in the astronomical unit of $15 \pm 4 \mathrm{~m} /$ cyr has been cited by Krasinsky et al. [15] Although planetary observations contain many possible sources of error, the apparent change is of similar order of magnitude to the lunar orbit anomaly. The discrepancy in AU could also be partially due to change in $c$. On the Moon we have the advantage of laser reflectors, providing a more precise standard of measurement.

\section{Findings}

Comparison of the Lunar Laser Ranging Experiment with independent datasets shows a very significant anomaly in LLRE measurements of lunar orbital evolution. Independent measurements agree on a slower recession rate. Since LLRE alone relies on the speed of laser light, the anomaly may be precisely predicted by a timevariation in $\mathrm{c}$.

\section{Conclusions}

At one time scholars disagreed whether light travelled instantaneously or had a finite speed. Galileo suggested placing lanterns on distant hilltops to time light's passage, but lacked an accurate clock. Today we have laser lanterns and the distant hilltop of the Moon. Change in $c$ may be detectable in the apparent rate of lunar recession.

The puzzle of "accelerating" redshifts is one of the most interesting in Physics. Lower redshifts increase linearly with distance, indicating expansion of the Universe. Redshifts of distant Type Ia supernovae appear to increase non-linearly, leading to speculation about acceleration and repulsive energies. Since redshifts are related to the speed of light, secular change in $c$ may precisely explain the "accelerating" puzzle.

A changing speed of light has been subject of consideration since at least the first Lord Kelvin [16], recently by Moffat [17], Albrecht and Maguiejo [18]. Applying lunar data to cosmology may shine light on hypothetical "dark" energies. With improved clocks, as will be available on the International Space Station, experimenters will be able to measure $c$ precisely in the laboratory. As with Jupiter's moons and Mercury, anomalies in orbits may have significance for Physics.

This work was assisted by NASA and the Beyond Einstein program. Great thanks are due to colleagues at the ARES group in Johnson Space Center, Houston. Encouraging communications were made with Marni Dee Sheppeard and Yves-Henry Sanejouand. 


\section{Competing interests}

The author declare that they have no competing interests.

\section{Authors' contributions}

LR collected observations of the Moon and drafted the manuscript. The author has read and approved the final manuscript.

Received: 7 September 2011 Accepted: 8 March 2012

Published: 20 April 2012

\section{References}

1. Nordtvedt K: Post-Newtonian gravitational effects in lunar laser ranging. Phys Rev D 1973, 7:2347-2356.

2. Williams JG, Dicke RH, Bender PL, Alley CO, Carter WE, Currie DG, Eckhardt DH, Faller JE, Kaula WM, Mulholland JD, Plotkin HH, Poultney SK, Shelus PJ, Silverberg EC, Sinclair WS, Slace MA, Wilkinson DT: New test of the equivalence principle from lunar laser ranging. Phys Rev Lett 1976 , 36:551-554

3. Williams JG, Newhall XX, Dickey JO: Relativity parameters determined from lunar laser ranging. Phys Rev D 1996, 53:6730-6739.

4. Williams JG, Turyshev SG, Boggs DH: Progress in lunar laser ranging tests of relativistic gravity. Phys Rev Lett 2004, 93:261101-261104.

5. Battat JBR, Chandler JF, Stubbs CW: Testing for Lorentz violation: Constraints on standard-model-extension parameters via lunar laser ranging. Phys Rev Lett 2007, 99:241103-241106.

6. Dickey JO, Bender PL, Faller JE, Newhall XX, Ricklefs RL, Ries JG, Shelus PJ, Veillet C, Whipple AL, Wiant JR, Williams JG, Yoder CF: Lunar laser ranging: a continuing legacy of the Apollo program. Science 1994, 265:482-490.

7. Bills BG, Ray RD: Lunar orbital evolution: a synthesis of recent results. Geophy Res Lett 1999, 26:3045-3048.

8. Williams GE: Geological constraints on the Precambrian history of Earth's rotation and the Moon's orbit. Rev Geophys 2000, 38:37-59.

9. Stephenson FR, Morrison LV: Long-term fluctuations in the Earth's rotation: 700 BC to AD 1990. Phil Trans Royal Soc 1995, 351:165-202.

10. Poliakow E: Proc Int Astron Union 2005, 2004:445.

11. Stephenson FR, Morrison LV: Historical values of the Earth's clock error $\Delta T$ and the calculation of eclipses. J History Astron 2004, 35:327-336.

12. Riofrio L: In Observing Dark Energy, proceedings of conference. Edited by Tucson AZ, Lauer T, Wolff S. San Francisco: Astronomical Society of Pacific; 2005.

13. Iorio L: Solar System planetary tests of c/c. Gen Relativ Gravitation 2009, 42:199-208

14. Iorio L: On the anomalous secular increase in eccentricity of the orbit of the Moon. Mon Not Royal Astr Soc 2011, 415:1266-1275.

15. Krasinsky GA, Brumberg VA: Secular Increase of Astronomical Unit from Analysis of the Major Planet Motions and its Interpretation. Celestial Mech Dyn Astron 2004, 90:267-288.

16. Thomson W, Tait PG: Nat Philosophy 1874, 1:403.

17. Moffat J, Clayton M: Physics Letters B 1999, 460:3.

18. Albrecht A, Magueijo J: Phys Rev D 1999, 59:043516.

doi:10.1186/2191-2521-1-1

Cite this article as: Riofrio: Calculation of lunar orbit anomaly. Planetary science 2012 1:1.

\section{Submit your manuscript to a SpringerOpen ${ }^{\odot}$ journal and benefit from:}

- Convenient online submission

- Rigorous peer review

- Immediate publication on acceptance

- Open access: articles freely available online

- High visibility within the field

- Retaining the copyright to your article

Submit your next manuscript at springeropen.com 